\title{
A minimally-invasive serial cerebrospinal fluid sampling model in conscious Göttingen minipigs
}

\author{
Alessandra Bergadano*1, Eva Maria Amen², Björn Jacobsen², Sara Belli², Anthony \\ Vandjour $^{2}$, Christelle Rapp ${ }^{2}$, Claudia Senn ${ }^{2}$. \\ ${ }^{1}$ Department for BioMedical Research, University of Bern \\ ${ }^{2}$ Roche Pharma Research and Early Development, Pharmaceutical Sciences, Roche Innovation Center \\ Basel, F. Hoffmann-La Roche Ltd, Grenzacherstrasse 124, 4070 Basel, Switzerland
}

\section{Corresponding author:}

\author{
Alessandra Bergadano \\ PD, DVM, Dr.Med.Vet., Dip.ECVAA, Dip. ECLAM, PhD \\ Head of Central Animal Facilities \\ Department for BioMedical Research, University of Bern \\ Murtenstrasse 35 \\ CH-3008 Bern \\ +41316328749 \\ +41792382393 \\ alessandra.bergadano@dbmr.unibe.ch
}

\section{Supplementary information - Table of content:}

- Table S1: Individual and average PK raw data obtained in minipig plasma and CSF 


\section{Supplementary information}

\begin{tabular}{|c|c|c|c|c|c|c|c|c|c|c|}
\hline \multirow[b]{3}{*}{ Route } & \multirow[b]{3}{*}{$\begin{array}{l}\text { Dose* } \\
(\mathrm{mg} / \mathrm{kg})\end{array}$} & \multirow[b]{3}{*}{ Matrix } & \multirow[b]{3}{*}{$\begin{array}{c}\text { Sampling } \\
\text { Site }\end{array}$} & \multirow[b]{3}{*}{$\begin{array}{l}\text { Hour } \\
\text { (h) }\end{array}$} & \multicolumn{3}{|c|}{ Subject } & \multirow{3}{*}{$\mathbf{N}$} & \multirow{3}{*}{ Mean } & \multirow{3}{*}{ SD } \\
\hline & & & & & 220613 & 222445 & 219514 & & & \\
\hline & & & & & \multicolumn{3}{|c|}{$\begin{array}{c}\text { Concentration } \\
(\mathrm{ng} / \mathrm{mL})\end{array}$} & & & \\
\hline $\begin{array}{l}\text { Subcutan } \\
\text { eous }\end{array}$ & 4.2 & $\begin{array}{c}\text { Plasm } \\
\text { a }\end{array}$ & Ear vein & predose & $\begin{array}{c}\text { no } \\
\text { compound }\end{array}$ & $\begin{array}{c}\text { no } \\
\text { compound }\end{array}$ & $\begin{array}{c}\text { no } \\
\text { compound }\end{array}$ & 3 & 0.00 & $\mathrm{NC}$ \\
\hline & & & & 0.5 & 5040 & 4640 & 4370 & 3 & 4680 & 337 \\
\hline & & & & 1.5 & 4670 & 4610 & 3590 & 3 & 4290 & 607 \\
\hline & & & & 3 & 2070 & 2500 & 1810 & 3 & 2130 & 348 \\
\hline & & & & 6 & 704 & 958 & 358 & 3 & 673 & 301 \\
\hline & & & & 24 & 49.3 & 17.1 & 11.6 & 3 & 26.0 & 20.4 \\
\hline \multicolumn{4}{|c|}{$25.5 \mathrm{~h}$ from study start or $1.5 \mathrm{~h}$ after last dose on day 2} & 25.5 & 3850 & 4730 & 3480 & 3 & 4020 & 642 \\
\hline
\end{tabular}

\begin{tabular}{|c|c|c|c|c|c|c|c|c|c|c|}
\hline \multirow[b]{3}{*}{ Route } & \multirow[b]{3}{*}{$\begin{array}{c}\text { Dose } \\
(\mathrm{mg} / \mathrm{kg})\end{array}$} & \multirow[b]{3}{*}{ Matrix } & \multirow[b]{3}{*}{$\begin{array}{c}\text { Sampling } \\
\text { Site }\end{array}$} & \multirow[b]{3}{*}{$\begin{array}{c}\text { Hour } \\
\text { (h) }\end{array}$} & \multicolumn{3}{|c|}{ Subject } & \multirow[b]{2}{*}{$\mathbf{N}$} & \multirow[b]{2}{*}{ Mean* } & \multirow[b]{2}{*}{ SD } \\
\hline & & & & & 220613 & 222445 & 219514 & & & \\
\hline & & & & & \multicolumn{6}{|c|}{$\begin{array}{c}\text { Concentration } \\
(\mathrm{ng} / \mathrm{mL})\end{array}$} \\
\hline $\begin{array}{l}\text { Subcutan } \\
\text { eous }\end{array}$ & 4.2 & CSF & Lumbar & predose & $\begin{array}{c}\text { no } \\
\text { compound }\end{array}$ & $\begin{array}{c}\text { no } \\
\text { compound }\end{array}$ & $\begin{array}{c}\text { no } \\
\text { compound }\end{array}$ & 3 & 0.00 & NC \\
\hline & & & & 0.5 & 25.2 & $\leq 10$ & $\leq 10$ & 3 & 15.1 & 8.8 \\
\hline & & & & 1.5 & 34.0 & 32.4 & 25.9 & 3 & 30.8 & 4.3 \\
\hline & & & & 3 & 19.6 & 57.5 & 30.2 & 3 & 35.8 & 19.6 \\
\hline & & & & 6 & $\leq 10$ & 43.7 & $\leq 10$ & 3 & 21.2 & 19.5 \\
\hline & & & & 24 & $\leq 10$ & $\leq 10$ & $\leq 10$ & 3 & $\leq 10$ & NC \\
\hline \multicolumn{4}{|c|}{$25.5 \mathrm{~h}$ from study start or $1.5 \mathrm{~h}$ after last dose on day 2} & 25.5 & 57.7 & 20.5 & 36.1 & 3 & 38.1 & 18.7 \\
\hline \multicolumn{4}{|c|}{ Cisterna Magna } & 25.5 & 48.0 & & & & & \\
\hline
\end{tabular}

Table S1: Individual and average PK raw data obtained in minipig plasma and CSF. on Day 1 and on Day 2, with a dosing interval of 24 hours; *For mean value calculations, concentration values $\leq \mathrm{LLOQ}$ were set as $=10 \mathrm{ng} / \mathrm{mL} ; \mathrm{SD}$, standard deviation; $\mathrm{NC}=$ not calculated 